MONTOMOLI F, MASSINI M, YANG H, HAN JC. (2012) 'THE BENEFIT OF HIGHCONDUCTIVITY MATERIALS IN FILM COOLED TURBINE NOZZLES'. INTERNATIONAL JOURNAL OF HEAT AND FLUID FLOW, 34, PP. 107-116

DOI: 10.1016/J.IJHEATFLUIDFLOW.2011.12.005

\title{
THE BENEFIT OF HIGH-CONDUCTIVITY MATERIALS IN FILM COOLED TURBINE NOZZLES
}

\author{
${ }^{1,2}$ F. Montomoli, ${ }^{1}$ M. Massini, ${ }^{3}$ H. Yang, ${ }^{3}$ J.C. Han \\ ${ }^{1}$ Whittle Laboratory, University of Cambridge, UK, ${ }^{2}$ BCAM, Spain \\ ${ }^{3}$ Turbine Heat Transfer Laboratory, Texas AM University, US
}

\section{ABSTRACT}

This study presents an experimental and numerical investigation of the beneficial effect of higher conductivity materials in HP turbine nozzles. Most of the literature studies focus on the maximum temperature that a nozzle can withstand, whereas the effect of thermal gradients is often neglected. However thermal gradients have higher influence on the life of the components and they have to be given careful consideration. In this work it is shown that thermal gradients are reduced by using high conductivity materials and, as a consequence, the nozzles life is appreciably increased.

A representative film cooled leading edge with an internal impingement plate was studied experimentally at Texas AM University. Two materials were used, namely polycarbonate and stainless steel, in order to highlight the impact of conduction on coolant effectiveness. Numerically conjugate heat transfer simulations have been carried out with an in house solver to analyse in detail the impact of conduction and internal convection. Both experimental and numerical results show that by increasing the conductivity in the solid region, the thermal gradients are strongly reduced. Numerically it is shown that using inserts of nickel-aluminide alloys in nozzles may reduce the thermal gradients from 3 to 4 times if compared to nowadays design.

\section{INTRODUCTION}

In gas turbine for power generation and mechanical drive, reliability is the key parameter. As shown by Fadlun et al. [1] the operating point, fuel composition, and other macro-parameters are subject to variations that influence performances and reliability. Among these parameters, two are mainly affecting the life of high-pressure nozzles: the maximum temperature and the metal thermal gradients.

Brandt and Wesorick [2] point out that most of nozzle failures are due to thermal fatigue mainly, whereas the absolute high temperature level has a lower impact. Moreover they show that the conditions of maximum temperature and maximum gradients happen at two different operating conditions. 
A similar conclusion has been obtained by Mazur et al. [4] analysing the failure of a high pressure nozzle of a $70 \mathrm{MW}$ gas turbine. The authors identified the origin of the cracks in nozzles by using numerical analysis and investigating the metal grain alterations. The critical region was identified to be the inner part of the coolant holes, corresponding to the highest concentration of thermal stresses.

This effect is particularly evident in nowadays design, which induce higher thermal stresses. As discussed by Bunker [5] the improvement of turbine cooling allowed higher maximum temperatures than in the past, but the increment in the system complexity increased the in-plane and throughwall thermal gradients. As shown by Eifel et al. [6] and Verstraete et al. [7] these thermal gradients can be reduced using conjugate heat transfer analysis during coolant optimization and must be used to predict maximum temperature and thermal stress when an accurate life prediction is required. Verstraete et al. [7], during the optimization process of a rotor blade, using as objective function the maximum temperature and the thermal gradients in the metal was able to obtain a significatnt improvement of blade life. Despite that, most of the studies presented in literature focus on the reduction of the effects of the maximum temperature rather than in controlling the thermal gradients.

One of the first papers identifying a possible solution for controlling the high thermal gradients in nozzles was written by Tret'yachenko et al. [3]. Working on marine propulsion applications; the authors were looking for a method to improve the life of nozzles with salty air. Moreover the nozzles were affected by strong thermal cycles, with frequent start-ups and shutdowns. One of the proposed solutions was using aluminium coating around the nozzle. The authors found an increment of nozzle life, with and without salt. The increment without salt was more than $40 \%$, due to the fact that aluminium was mitigating the thermal stresses.

In this work the analysis of coolant effectiveness on leading edge made in different materials is presented. A combination of two cooling technology, impingement and film cooling, is applied on a cylinder representative of modern gas turbine leading edges. A strong variation of effectiveness associated to the different materials is observed both experimentally and numerically. By introducing a non dimensional parameter for the temperature gradients, is possible to predict the impact of the material in a real gas turbine. It is shown numerically that using nickel-aluminide alloy for the leading edge reduces the metal gradients by 3-4 times the nowadays values. Moreover a reduction of $20 \%$ of thermal stresses for the thermal barrier coatings is predicted with reduced risk of TBC spallation.

\section{NOMENCLATURE}

d Cooling holes diameter $[\mathrm{m}]$

I Cooling holes length [m]

H Heat transfer coefficient [W/m2K]

$\mathrm{N} \quad$ Face normal

k Thermal conductivity [W/Km] 
Z Spanwise coordinate of the cylinder [m]

$\Delta \mathrm{n}_{\mathrm{s}} \quad$ Distance centre of the cell - wall (solid) [m]

$\Delta \mathrm{n}_{\mathrm{f}} \quad$ Distance centre of the cell - the wall (fluid) [m]

B Distance impingement plate-internal cylinder [m]

D Cylinder diameter [m]

$\mathrm{H} \quad$ Height of the cylinder [m]

L Thickness [m]

M Blowing ratio $\frac{\rho_{c} V_{c}}{\rho_{m} V_{m}}[-]$

Ma Mach number [-]

P Coolant pitch distance $[\mathrm{m}]$

$\mathrm{R} \quad$ Thermal resistance $\frac{\Delta T}{\text { heat flux }}[\mathrm{K} / \mathrm{W}]$

$\mathrm{T} \quad$ Temperature $[\mathrm{K}]$

V Velocity $[\mathrm{m} / \mathrm{s}]$

BC Bond coat

TBC Thermal barrier coating

NDG Non dimensional gradients $N D G=\frac{\partial T}{\partial z} \frac{D}{T_{t, m}-T_{t c}}$

$\theta \quad$ Tangential angle (0 at leading edge) [deg]

$\rho \quad$ Density

$\Phi$

Overall Coolant Effectiveness $\phi=\frac{T_{t, m}-T}{T_{t, m}-T_{t c}}$

Subscripts

c Coolant

a $\quad$ Adiabatic

m Main 
f $\quad$ Fluid

S Solid

tbc Thermal barrier coating

bc Bond coat

w Wall

\section{EXPERIMENTAL APPARATUS}

The test case presented in this paper represents a film cooled leading edge with internal impingement. A schematic of the rig is shown in fig. 1. The blow down wind tunnel, shown in fig.1a, used for this investigation, was also used previously by Ekkad et al. [10] and Gao et al. [11] with a similar layout. In fig 1 is shown the raw picture with wide band liquid crystal and the location of the coolant reference thermocouple.
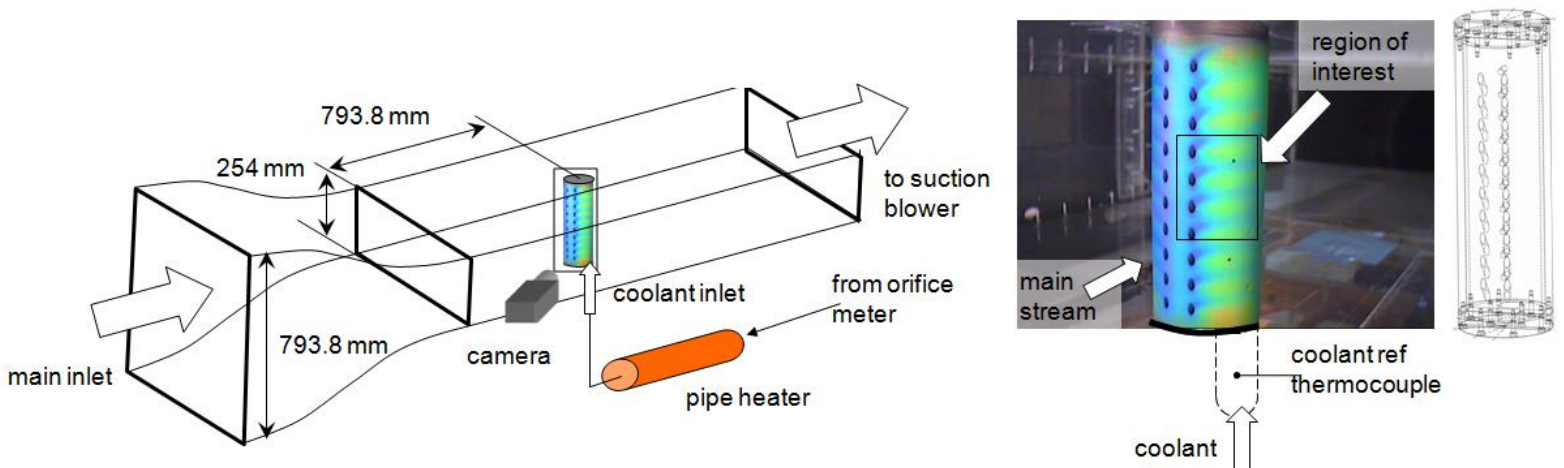

Fig 1 Schematic of blow down wind tunnel

Air at atmospheric condition is entering the tunnel through a convergent duct. The free stream turbulence level is below $1 \%$. The geometry studied is a cylinder with two rows of coolant holes and an impingement plate, as shown in the schematic of fig. 2 . Figure 2 shows a cut-view of the cylinder. The cylinder has a diameter of $76.2 \mathrm{~mm}$, equivalent to a Reynolds number based on free stream velocity of 100,900 . Two rows of 10 holes are located at \pm 15 degrees from the leading edge with a spanwise inclination of 30 degrees and a streamwise direction of 90 degrees. The main body of the cylinder is shown in fig. 2 . The hole diameter for film cooling is $d=4.75 \mathrm{~mm}$ with a spanwise distance between adjacent holes of $P / d=4$. The thickness of the cylinder shell is $6.5 \mathrm{~mm}$. The impingement plate has a thickness of $13 \mathrm{~mm}$. The length of the coolant holes $\mathrm{l} / \mathrm{d}=2.65$ is representative of the values currently used in heavy duty gas turbines at leading edges, from 2.5 to 5 .

A perforated plate is inserted internally, separating two cavities to model an internal impingement. The impingement plate is perforated by 10 holes with the same diameter $\mathrm{d}$ of the cooling holes. The 
impingement holes are axial. Having 10 holes for the impingement and 20 for the film cooling, a velocity ratio of approximately 2 is obtained between the impingement jets and coolant holes. The distance between the holes and the internal surface is $B / d=5.3$. In real gas turbines $B / d$ is in a range between 4.5 and 6 .

In order to measure the coolant effectiveness a temperature gradient is required between the coolant and the main stream. As common practice in the open literature (Coat and Lock [12], Teng et al. [13]) the coolant flow is heated up to a temperature which differ from the main stream of about 30 degrees. During these tests the "coolant" is warmed up to temperature between 330K and 405K by a pipe heater, fig. 1, before entering the cylinder. The blowing ratio of these configurations is the same of a real gas turbine. By using this technique the density ratio is not fully matched if the coolant fluid is air. Ethridge et al [14] have pointed out that the impact of density ratio near the leading edge is more important for values of blowing ratio below 0.3 . In this work all the data presented are for higher values of blowing ratio. However Ekkad et al. [10] showed that the effect of density ratio is not negligible for the effectiveness. They studied the impact of density ratio on the same configuration investigated in this work, with pure film cooling and found that there were almost no differences in the Nusselt number distribution. Nevertheless some differences were found for the effectiveness distribution. By using a density ratio, DR=1.5 (gas $\mathrm{CO}_{2}$ ) the maximum effectiveness was found for $M=0.8$. By using air with $D R=1.0$ instead, the effectiveness incraeses continuously from $M=0.4$ to 1.2 . In this work air is used and a similar trend is expected. Despite these differences on the effectiveness, the impact of conduction on metal gradients is expected to be the same.

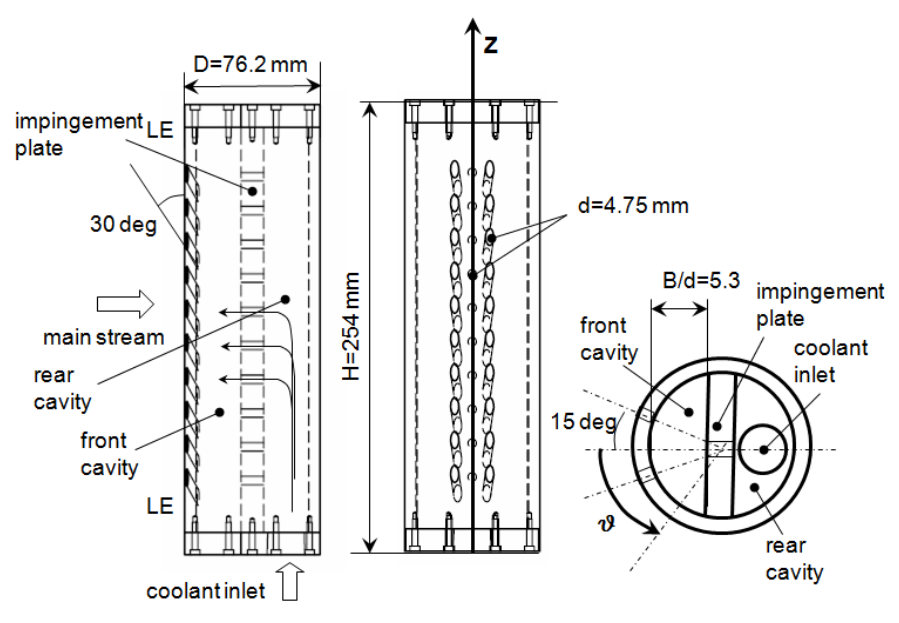

Fig 2 Geometry of film cooling leading edge

In order to evaluate the effect of thermal conductivity on conjugate heat transfer, two geometries have been built using polycarbonate $(k=0.2 \mathrm{~W} / \mathrm{mK})$ and stainless steel $(k=16.18 \mathrm{~W} / \mathrm{mK})$.

A detailed analysis of different visualization techniques on a similar configuration, without impingement, has been carried out by Gao et al. [11]. During this experimental campaign steady IR measurements and wide band liquid crystals have been used and the agreement was satisfactory. In the present paper only the wide band liquid crystals results will be presented. 
Three thermocouples have been placed inside the rear cavity in order to measure the temperature distribution of the flow at different operating points. Figure 3 shows the radial distribution of temperature for $M=0.4,0.8,1.2$ for the stainless steel test. The test case shows a small reduction of coolant temperature in the rear cavity moving towards the top. For this reason the complete model has been simulated in the numerical simulation.

Table 1 shows the measured temperature of coolant and of the main stream for different operating conditions and materials. Two different configurations have been investigated: pure impingement and combination of film cooling and impingement, fig.4.

In the configuration of pure impingement the cylinder was rotated by 180 deg and the coolant holes were sealed. The coolant was entering from the same inlet and it was coming out from the front cavity through two slots located at both ends of the cylinder. The configurations with a combination of film cooling and impingement, and pure impingement with the same blowing ratio have been tested with the same coolant mass flow. In this way the velocity of the impinging jet is the same for the same nominal blowing ratio.

Analyzing the heat transfer with impingement alone and combination of film cooling and impingement is possible to understand qualitatively the contribution of both to the combined cooling.

The results discussed in this paper are on the combination of film cooling and impingement, since the pure film cooling case is already discussed in Gao et al. [11]. The coolant temperature has been measured in the pipe feeding the rear cavity placed $2 \mathrm{~cm}$ below the cylinder base. The coolant mass flow was measured using an orifice flow meter, fig. 1.

\section{COMPUTATIONAL METHODOLOGY}

The numerical simulations were carried out on the same configuration analysed experimentally.

\section{CFD SOLVER}

The CFD simulations have been carried out using an in house CFD solver Hybflow, by Adami et al. [15] and Montomoli et al. [16] developed at the University of Florence. The spatial discretisation is based on a finite volume approach for hybrid unstructured grids. The Roe's approximate method is used for the up-winding of convective fluxes. A linear reconstruction of the solution inside the elements provides a second order discretisation while monotonicity is ensured through the TVD slope limiter proposed by Barth [17]. Turbulent viscous flows are represented by the conventional mass-averaged system of Navier-Stokes equations with the eddy viscosity determined according to the two equations $k-\omega$ turbulent model proposed by Wilcox, [18] with realizability constraint of Durbin.

The CFD solver HybFlow, with the same turbulence closure used in this work, has been extensively validated for film cooling simulations [20]. In particular a previous study in a film cooled leading edge shows that the kidneys vortices obtained with the CFD simulations and the experimental results with hot wire measurements are in a good agreement in terms of vorticity and turbulence levels. The 
standard k- $\omega$ model of Wilcox is used in literature as one of the most accurate two equations models to predict film cooling structures (Harrison K.L. and Bogard D. G. [19]). The CFD solver HybFlow, with this turbulence closures, has been used to predict the coolant effectiveness and discharge coefficient in real conditions with and without internal cross flows [21].

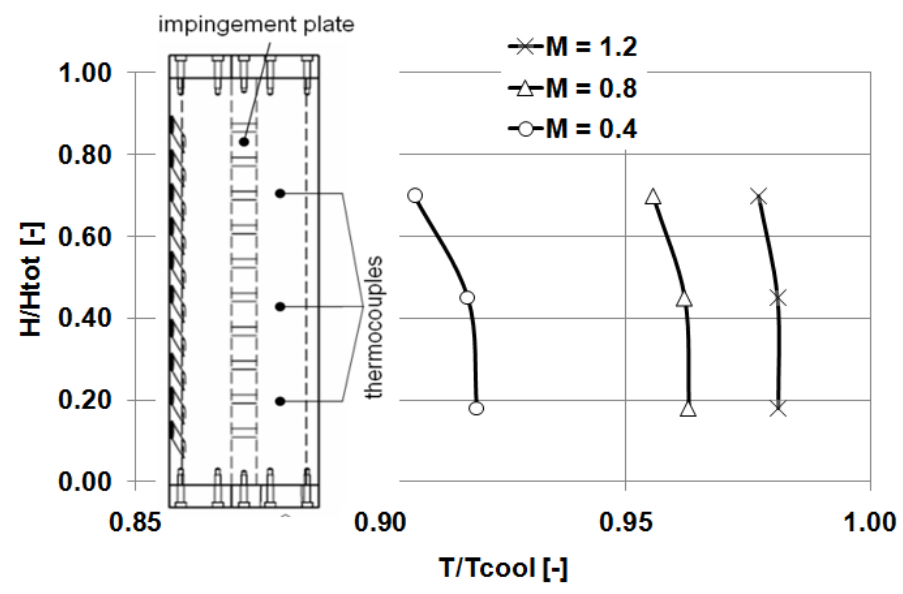

Fig 3 Temperature distribution in the rear cavity with stainless steel cylinder
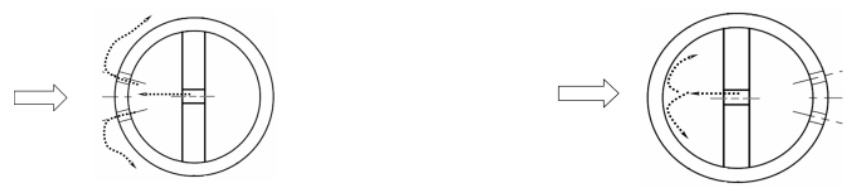

Fig. 4 combination of film cooling and impingement and pure impingement

\begin{tabular}{cc|cc|cc} 
& \multicolumn{2}{c|}{ Polycarbonate } & \multicolumn{2}{c}{ Stainless steel } \\
\cline { 3 - 6 }$M$ & \multicolumn{2}{c}{ Combination } & Impingement & Combination & Impingement \\
\hline 0.4 & $\mathrm{~T}_{\mathrm{c}}$ & $378[\mathrm{~K}]$ & $405[\mathrm{~K}]$ & $374[\mathrm{~K}]$ & $380[\mathrm{~K}]$ \\
& $\mathrm{T}_{m}$ & $300[\mathrm{~K}]$ & $301[\mathrm{~K}]$ & $300[\mathrm{~K}]$ & $301[\mathrm{~K}]$ \\
\cline { 2 - 6 } 0.8 & $\mathrm{~T}_{\mathrm{c}}$ & $362[\mathrm{~K}]$ & $364[\mathrm{~K}]$ & $346[\mathrm{~K}]$ & $355[\mathrm{~K}]$ \\
& $\mathrm{T}_{m}$ & $301[\mathrm{~K}]$ & $301[\mathrm{~K}]$ & $301[\mathrm{~K}]$ & $301[\mathrm{~K}]$ \\
\cline { 2 - 6 } 1.2 & $\mathrm{~T}_{\mathrm{c}}$ & $349[\mathrm{~K}]$ & $343[\mathrm{~K}]$ & $331[\mathrm{~K}]$ & $331[\mathrm{~K}]$ \\
& $\mathrm{T}_{m}$ & $302[\mathrm{~K}]$ & $303[\mathrm{~K}]$ & $302[\mathrm{~K}]$ & $302[\mathrm{~K}]$ \\
\cline { 2 - 5 } & \multicolumn{2}{c}{} & \multicolumn{2}{c}{}
\end{tabular}

Tab 1 Operative test matrix

The conjugate heat transfer simulation has been performed with a Fourier solver integrated in the code. A more detailed description of the code can be found in Montomoli et al. [16]. The heat transfer Fourier's equation for the solid zone is evaluated through an explicit time marching scheme. The coupling procedure implemented is a standard temperature forward flux back method (Verstraete [22]). In this particular method the stability is not dependent on the Biot number if the temperature at the interface is updated every iteration, as in the present implementation. The conjugate heat transfer capability of the solver has been previously validated [16] using the analytical solution of Luikov for a flat plate [25] and on a film cooled surface (Martiny et al. [26]), not shown in this work. 
The computational grid has been obtained using the commercial grid generator Centaur. The mesh is an unstructured hybrid grid with prismatic layers close to the walls and tetrahedrons in the fluid core. The anisotropic prismatic mesh at the solid wall allows a different clustering level along and normal to the wall. The solid geometry is tessellated using tetrahedrons only. A detailed view of the mesh is shown in fig 5 . The fluid solid interface is studied using conformal meshes with hanging nodes. A symmetry plane is used in order to save computational resources.

A mesh dependency study on a similar layout has been carried out by Gao et al. [23]. The authors have found that about 1 million elements are needed for a single coolant hole. In this study 12 million elements have been used for the fluid domain and about 2 million for the solid one. The prismatic layer to analyse the near wall region is made of 15 layers with 1.25 aspect ratio. The value of $y+$ on the external surface of the cylinder is as avarage 0.7 for $\theta=90$ deg (where the value of $y+$ is maximum). The sublayer has been discretized by using 4 prismatic cells for $y+<5$.

A grid sensitivity analysis has been carried out applying the Richardson's extrapolation criterion. Two parameters have been used to evaluate the grid independency: the coolant mass flow and the surface adiabatic effectiveness. Three meshes have been built with 4, 10 and 12 million of elements for the fluid region. No differences have been observed in the solution between 10 and 12 million elements. The variation of coolant mass flow and the local coolant effectiveness was less than 0.1 percent between the two grids of 10 and 12 million elements.

\section{PARAMETERS USED FOR THE COMPARISON}

The main parameter used for the experimental analysis is the overall coolant effectiveness defined as:

$$
\phi=\frac{T_{t m}-T}{T_{t m}-T_{t c}}
$$

A value of $\phi=1$ represents a perfect film cooling performance, when the surface temperature is equal to the coolant temperature; a value of $\phi=0$ means that the film cooling has no effect in reducing the wall temperature, which is heated to the same temperature as the mainstream gas. In this study both conduction and convection contribute to modify the coolant effectiveness distribution. The model has been designed to operate at realistic Reynolds numbers. 


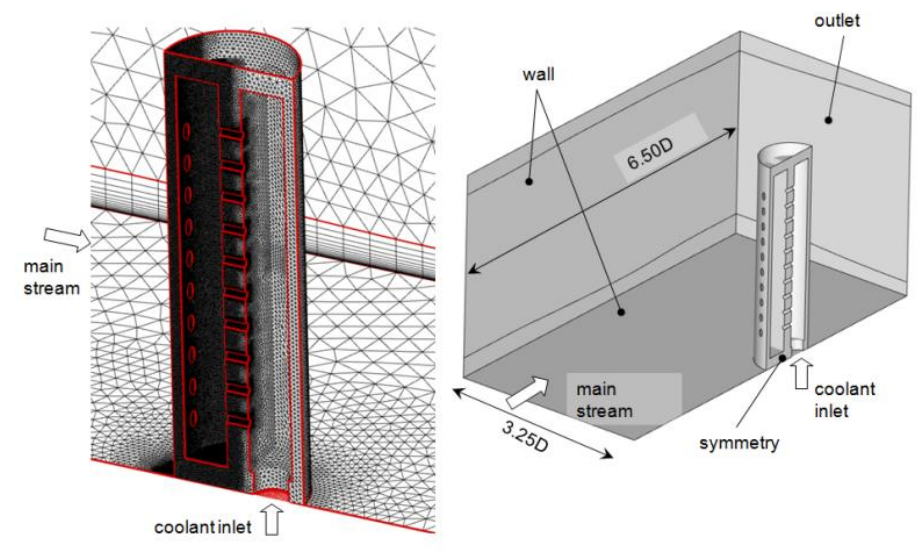

Fig 5 grid and computational domain

The uncertainty in the coolant and mainstream temperatures is $0.5^{\circ} \mathrm{C}$. The uncertainty of the overall coolant effectiveness can be evaluated with the methodology proposed by Kline and McClintock. By applying the chain rule the maximum error is between $7 \%$ (for $\phi=1$ ) and $5 \%$ (for $\phi=0$ ). This is obtained by:

$$
\varepsilon^{M A X}=\frac{\varepsilon_{T_{t}}}{T_{c}-T_{t}} \sqrt{\varphi^{2}+1}
$$

Where $\varepsilon^{M A X}$ is the maximum error of the overall coolant effectiveness and $\varepsilon_{T_{t}}$ is the uncertainty in the mainstream temperature.

The heat transfer coefficient in the front part of the cylinder is representative of the conditions found in a real gas turbine. The Biot number of the configuration used in this work was evaluated and compared with the Biot number of a real gas turbine [28]. The studied geometry represents a heavy duty gas turbine for power generation.

The thermal conductivity of the metal used for the first nozzle is about $k_{m}=16 \mathrm{~W} / \mathrm{mK}$. The resulting Reynolds number, based on leading edge diameter, is $R_{D}=100.900$ at $1800 \mathrm{~K}$ as turbine entry temperature. The heat transfer load can be obtained by $\mathrm{Nu} / \mathrm{Re}^{0.5}=1.6$ [28], and consequently the Nusselt number is $\mathrm{Nu}=564$. Considering the thermal conduction of air at $1800 \mathrm{~K}$ equal to $\mathrm{k}_{\mathrm{f}}=0.107$, the resulting Biot number is 0.2 , representative of heavy duty turbines. The value of the Biot number in high pressure nozzles for aeronautical propulsion instead is much higher and of the order of 1-1.8 [28]. The geometrical configuration proposed in this work has a Biot number of 0.1 for the stainless steel case and 6 for the polycarbonate case at design condition. The configuration with stainless steel has a similar Biot number of a real leading edge for heavy duty configuration without thermal barrier coating. With TBCs the solution is between the polycarbonate case and the stainless steel one.

In order to evaluate the thermal gradients in the real turbine a non dimensional group for the gradients has been introduced, defined as NDG (non dimensional gradients) 
$N D G=\frac{\partial T}{\partial z} \frac{D}{T_{t, m}-T_{t c}}$

Introducing this group on the present results the corresponding thermal gradients in the real case can be evaluated. The NDG is the local thermal gradients, $\delta T / \delta z$, scaled using $D /\left(T_{t m}-T_{t c}\right)$. The ratio $\mathrm{D} /\left(\mathrm{T}_{\mathrm{tm}}-\mathrm{T}_{\mathrm{tc}}\right)$ is twice the radial thermal gradient that is present in a solid cylinder, having the external surface at the same temperature of the main stream and the centre at the coolant one. The operative conditions of the present test case are representative of the real film effectiveness in a gas turbine. The scaling parameter for the effectiveness is the difference between hot gas temperature and film cooling, $T_{\mathrm{tm}}-T_{\mathrm{tc}}$. Moreover the geometry is scaled maintaining the same Reynolds number of real configurations.

The uncertainty for the NDG value can be obtained with the same methodology shown for the overall coolant effectiveness. In this case the expression becomes:

$$
\varepsilon_{N D G}^{M A X}=\frac{\varepsilon_{T_{t}}}{T_{c}-T_{t}} \sqrt{2 \varphi^{2}+1}
$$

For the NDG the maximum error is $8 \%$ (for $\phi=1$ ) and $5 \%$ (for $\phi=0$ )

All the geometrical parameters, $\mathrm{P} / \mathrm{d}, \mathrm{Z} / \mathrm{d}$, etc are the same of a real gas turbine. As a consequence the thermal gradients, in the non dimensional form proposed, are correctly scaled and can be correlated to the gradients found in a real gas turbine.

\section{EXPERIMENTAL RESULTS}

The experimental results presented show the conjugate heat transfer on the leading edge where both film cooling and impingement are applied.

The results of the experiments are depicted in Fig. 6, where the contour plots of cooling effectiveness are shown. The highest temperature, corresponding in the experimental set-up to the temperature of the coolant, indicates highest effectiveness. Three blowing ratios have been studied: $M=0.4,0.8$, 1.2. In Fig. 6 the results of the experiments are shown for the same cooling configuration. Two leading edges made in different materials are compared. In the left hand side of figure 6 the results for a leading edge made in polycarbonate are shown and in the right hand side the leading edge made in stainless steel are shown. The leading edge location corresponds to $\theta=0$ [deg], the impingement plate location is for $\theta=90$ [deg].

Polycarbonate
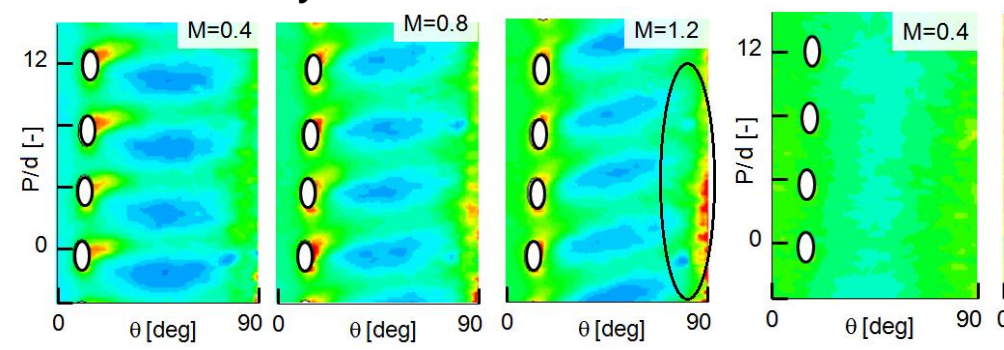

Stainless steel

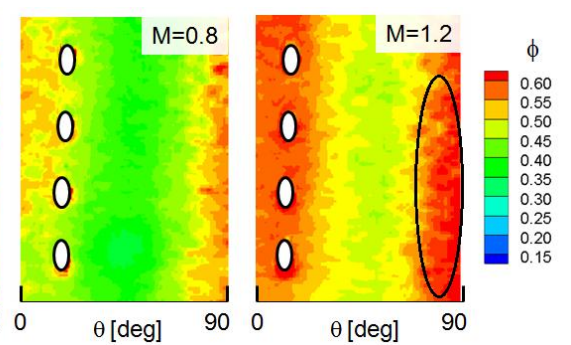

Fig. 6 Coolant effectiveness on the leading edge for $M=0.4,0.8,1.2$ and $k=0.2$ and $16.18 \mathrm{~W} / \mathrm{mK}$, film cooling and impingement, polycarbonate (left) and stainless steel (right) 

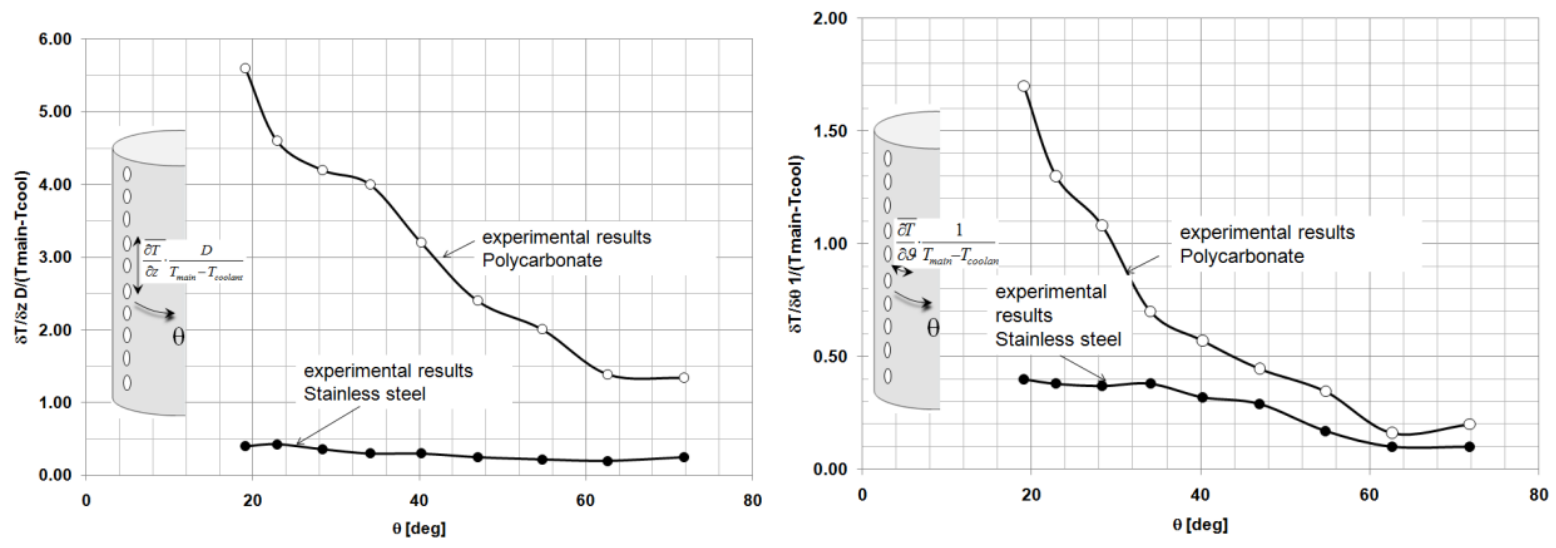

Fig. $7 \mathrm{NDG}$ comparison for $\mathrm{M}=1.2$ for $\mathrm{k}=0.2$ and $16.18 \mathrm{~W} / \mathrm{mK}$, film cooling and impingement, polycarbonate and stainless steel

The comparison of the results of Fig. 6 evidences that for all the blowing ratios, $0.4<M<1.2$, the thermal gradient on the surface are drastically reduced using a leading edge with higher thermal conductivity, i.e. the metallic model in this experiment. In particular the effectiveness is much more uniform both spanwise and tangentially.

The different behaviours of the materials could be predicted analysing the Biot number for the two cases. The Biot number, $\mathrm{Bi}=\mathrm{hL} / \mathrm{k}$, gives an indication on the ratio between the superficial heat transfer and the conduction coefficient of the solid. The Biot number of these experiments is respectively of the order of 0.1 for the metallic model and 6 for the plastic model. For a Biot number equal to 0.1 , the internal resistance to the heat transfer due to conduction is about $10 \%$ of the resistance of the surface to the heat exchange with the fluid. For low Biot number the local surface temperature is strongly affected by conduction. Therefore the thermal gradients, due to the direct effect of convection with the flow with high temperature gradients, are minimized. This is shown in fig 7 where the spanwise and tangential thermal gradients are compared for the polycarbonate case, white dots, and stainless steel one, black dots. It is evident that increasing the thermal conduction and consequently reducing the Biot number reduce the thermal gradients by 4-5 times. An interesting phenomenon can be noticed in Fig. 6 . At the position marked by the dashed ellipse (90 degrees from the leading edge) the effectiveness is higher than expected if compared to the results of Gao et al. [11]. The same phenomenon is observed in both the models, in plastic and in metal, and for all the blowing ratios. However the area affected by the high temperatures (and therefore, in these experiments, higher effectiveness) is more extended in the metallic model. 

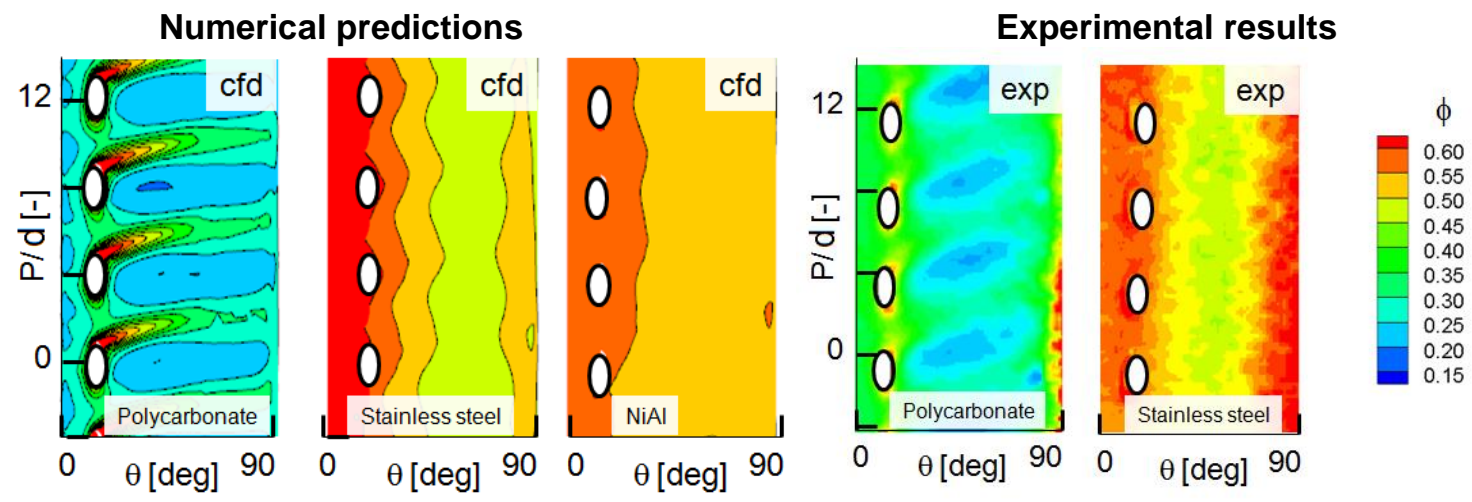

Fig. 9 Coolant effectiveness on the leading edge for $M=1.2$ and $k=0.2,16.18$ and $69 \mathrm{~W} / \mathrm{mK}$, film cooling and impingement
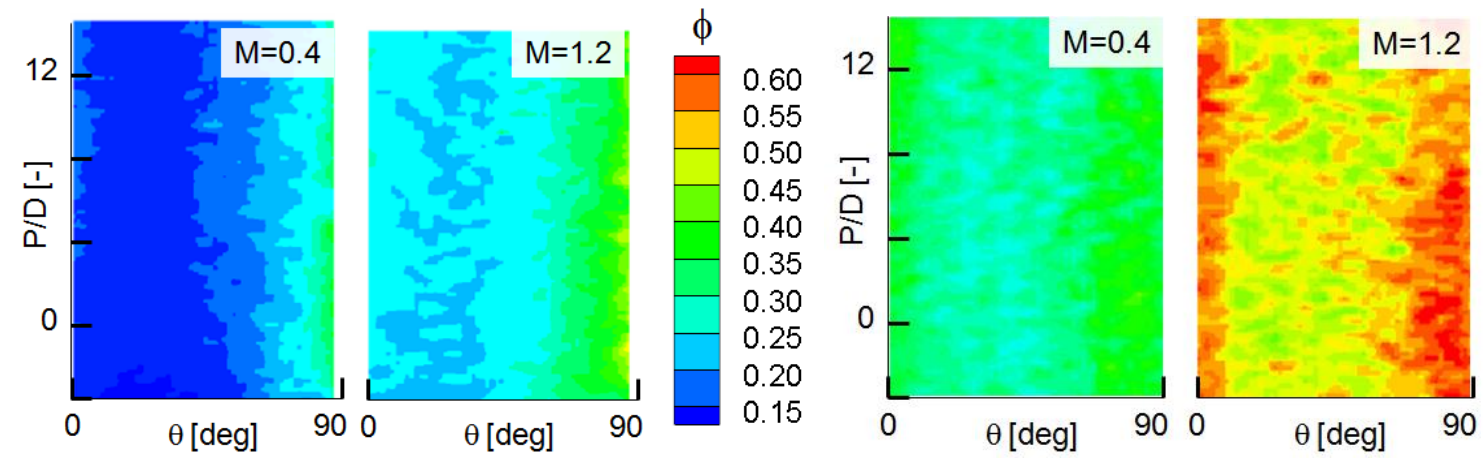

Fig. 8 Coolant effectiveness on the leading edge for $M=0.4,1.2$ and $k=0.2$ and $16.18 \mathrm{~W} / \mathrm{mK}$, pure impingement

In order to investigate better this aspect a configuration with only the impingement plate, without film cooling injection is studied. In this way it is possible to separate the film cooling component from the impingement one. The results of these tests are depicted in Fig. 8. In the left hand side of the figure the polycarbonate case is shown. In the right hand side the solution with stainless steel is presented.

Comparing the configurations in Fig. 8 with the configurations in Fig. 6, where also film cooling is included, it is clear that the high effectiveness at the location marked with the ellipse is generated by the impingement plate. The effectiveness is higher in the same location for the configuration with impingement only. This phenomenon is explained by the presence of the internal plate used for impingement. The heat exchange with the internal cooling flow is high due to conduction through the solid and it is higher for the material with higher thermal conductivity. The exploitation of this effect suggests the use of thermal bridges to minimize thermal gradients and optimize the cooling. The idea is using metal with high conductivity to extract heat locally and to reduce the thermal gradient.

As suggested by Bunker [5] it is advantageous for the life of the components aiming to isothermal conditions, to minimize all the stresses. Thermal bridges appear to be a possible method to achieve the minimization of thermal stresses and enhance the uniformity of surface temperature. 


\section{NUMERICAL RESULTS}

In order to quantify the heat transfer characteristics of the phenomenon, numerical simulations on the same cases have been carried out. The CFD results show the effect of a change in thermal conductivity of the leading edge material (Fig. 9). The thermal conductivity of the two materials analyzed changes of approximately 2 orders of magnitude passing from polycarbonate $(\mathrm{k}=0.2 \mathrm{~W} / \mathrm{mK})$ to stainless steel ( $\mathrm{k}=16 \mathrm{~W} / \mathrm{mK})$. On the right hand side of fig. 9 there are the experimental results for comparison. An extra numerical simulation was carried out in order to analyze the behaviour of a superalloy with good high temperature characteristic and higher thermal conductivity than standard Nickel superalloy. The chosen material for this analysis is a nickel-aluminide alloy, NiAl, having a thermal conductivity $\mathrm{k}=69 \mathrm{~W} / \mathrm{mK}$. The NiAl can be used as insert in regions of the nozzle where the thermal stresses are more important than the mechanical loadings, i.e. near the leading edge. The density of a NiAl alloy is $5.86 \mathrm{~g} / \mathrm{cm}^{3}$, about $30 \%$ less dense than a nickel alloy, $8.65 \mathrm{~g} / \mathrm{cm}^{3}$. This suggests a beneficial effect in terms of weight reduction. The case with NiAl has not been studied experimentally.

The solution for the polycarbonate case, shown in Fig. 9, has strong tangential and spanwise gradients, in agreement with what was observed in the experimental results. The thermal gradients are drastically reduced moving from the polycarbonate model to the stainless steel model and from the latter to the model in nickel-aluminide alloy. In particular the effect is evident in the area around the exit of the cooling holes. This area is the zone where the thermal stresses are critical and cracks are likely to occur, as highlighted by Mazur et al. [4].

The numerical simulations under predict the overall effectiveness. Numerically one of the issues related to a film cooled leading edge with RANS is the over-production of turbulence due to the stagnation point anomaly [31]. The code has been modified to avoid this problem but it seems under predicting the effectiveness of about $5-10 \%$.

The stainless steel case shows a second region with high effectiveness due to the presence of the impingement plate at $\theta=90$ degrees. The level of effectiveness reached by conduction is comparable to what is obtained near a film cooling exit. Moreover the coolant effectiveness is more uniform. The presence of the impingement plate is less evident than in the experimental results. This can be due to a slightly different value for the thermal conductivity in the experimental and numerical model but the overall trend for the reduction of thermal gradients is confirmed. As shown in $[29,30]$ the uncertainty of the metal conduction for stainless steel is greater than $5 \%$, for the range of temperatures studied in this work. An additional uncertainty is due to estimate of contact resistance between different components that are not been modelled here. Using the NiAl leading edge a general improvement in the lateral and tangential distributions of temperature is observed. The conduction generates a very uniform lateral distribution that is almost impossible to achieve near the leading edge region if only the design of the coolant system is changed.

In order to compare quantitatively the different materials the average effectiveness on the frontal side of the cylinder is computed and the non-dimensional temperature gradients on the main two directions, spanwise and tangential, are plotted. The average effectiveness of leading edge is computed area averaging the effectiveness of the frontal 15 degrees of the cylinder for $M=1.2$ (assuming symmetry respect the plane containing the axis of the cylinder and the direction of the flow). 
The average effectiveness for the frontal side of the cylinder is 0.25 for the polycarbonate model and 0.60 for the stainless steel model. Using a NiAl alloy the effectiveness is reduced to 0.57 if compared to the stainless steel case. The NiAL, having higher conductivity, spread the effect of internal impingement over a bigger area and this local reduction in effectiveness is negligible. There is a big advantage spreading the impact of impingement over a bigger surface and reducing the thermal gradients. As pointed out by Chernobrovkin and Lakshminarayana [24] the region immediately downstream the coolant holes can be very critical in case of a wrong design or off-design conditions. Using high conductivity material this risk is mitigated.
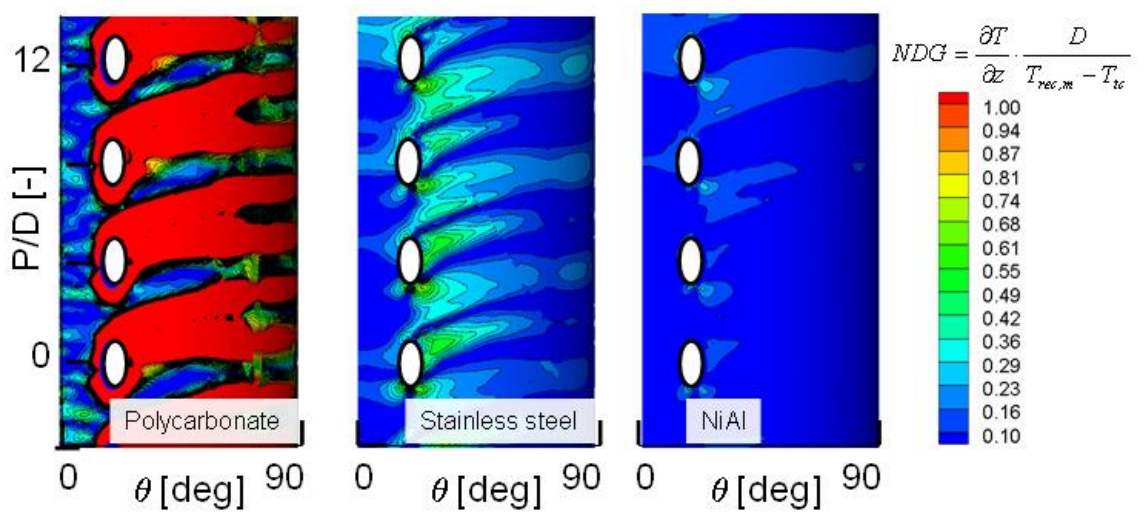

Fig 10 Spanwise NDG M=1.2 and $k=0.2,16.18$ and $69 \mathrm{~W} / \mathrm{mK}$, film cooling and impingement

Figure 10 shows the spanwise non dimensional gradients for $M=1.2$ and three materials. Moving from the polycarbonate case to the NiAl one, the thermal gradients are strongly reduced. In particular it is possible to observe that near the coolant holes there are the strongest gradients. The NDG reduction can be analysed comparing the tangential and spanwise non dimensional gradients along the profile. Both gradients are averaged in the spanwise direction using the absolute value of the gradient.

Figure 11 shows the comparison of spanwise gradients for stainless steel and NiAl. The $x$-axis is the tangential angle defined as zero at the leading edge and 90 at the impingement plate location. At 15 degrees are located the film cooling holes. The y-axis on the left indicates the mean NDG spanwise gradient. The absolute value of the gradient is averaged in the spanwise direction. On the right there is the difference between stainless steel and NiAI NDGs as NDGsteel/NDGNiAl percent. The dashed line corresponds to the NiAl case, the continuous black line to the stainless steel case and the red line is the percent difference. 


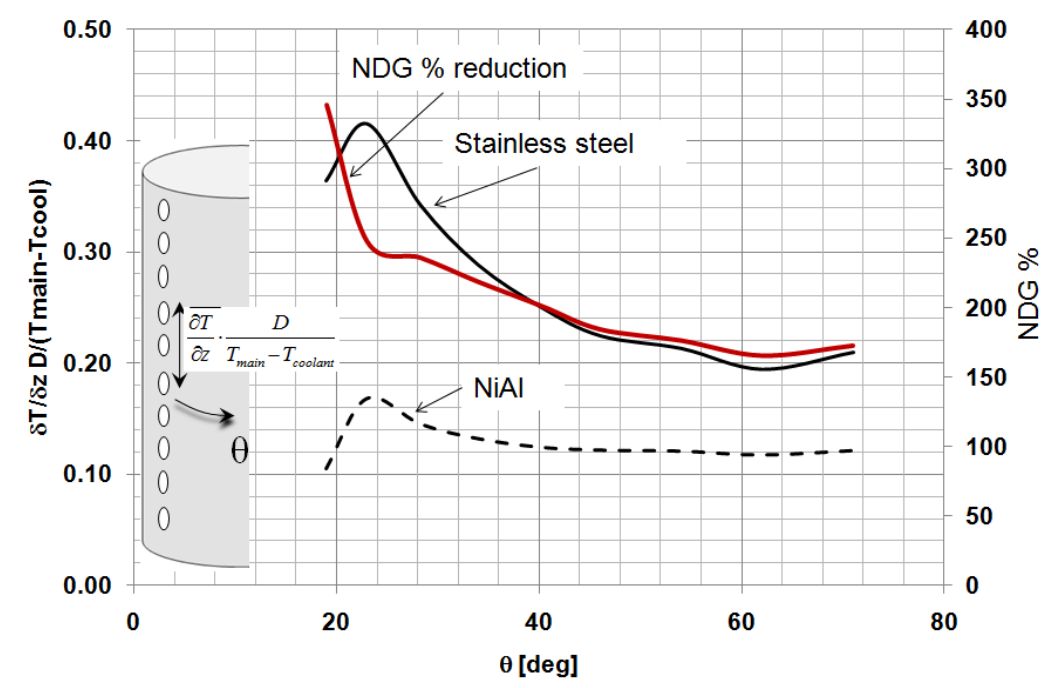

Fig. 11 mean spanwise NDG

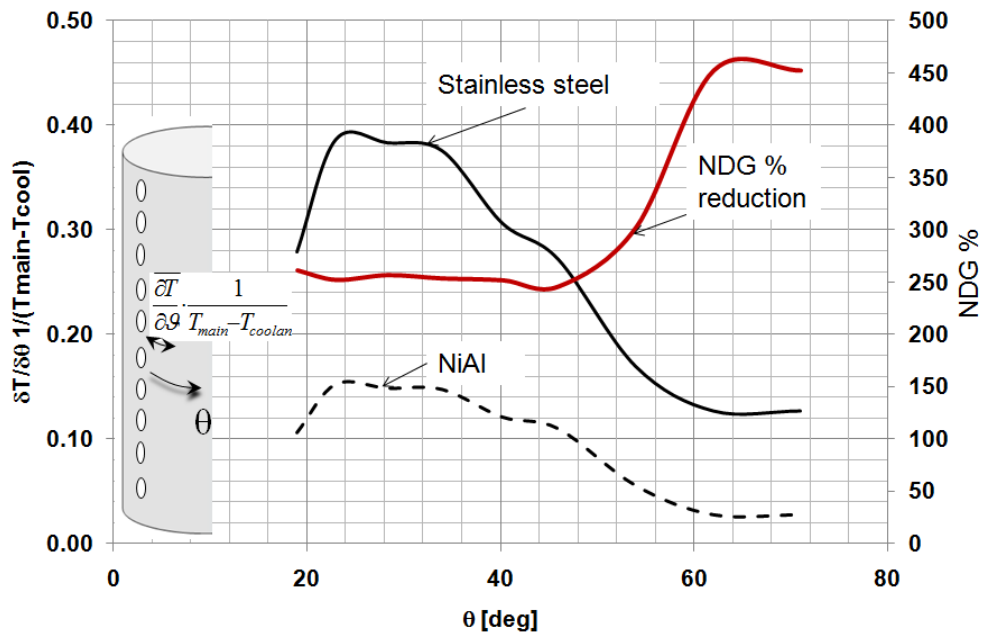

Fig. 12 mean tangential NDG

Near the leading edge there is the biggest difference of spanwise NDGs between the two configurations. In particular the gradients are reduced by about 3 times near the coolant holes. Moving towards the plate insert the spanwise NDGs are reduced of about 2 times.

Figure 12 shows the same graph in terms of tangential gradients. Near the leading edge the beneficial effect of having $\mathrm{n}$ higher conductivity material is still evident with a reduction of about 2.5 times. The biggest differences are moving towards 90 degrees where the effect of impingement plate is evident mitigating the tangential gradients. The results presented above are for the configuration without thermal barrier coating. In a real configuration the surface is covered by TBC and its contribution must be evaluated.

In this example a standard distribution of thermal barrier coating of Yttria Stabilized Zirconia (YSZ) is chosen for a real leading edge: $\mathrm{Y}_{2} \mathrm{O}_{3}$ stabilized with $\mathrm{ZrO}_{2}$ with thermal conductivity $\mathrm{k}=2.1 \mathrm{~W} / \mathrm{mK}$ in dense deposition and a nominal thickness of $300 \mu \mathrm{m}$. A bound coat of NiCoCrAlY is assumed with $\mathrm{k}=10.2 \mathrm{~W} / \mathrm{mK}$ and thickness of $100 \mu \mathrm{m}$. By assuming this distribution for a real leading edge an equivalent thickness is defined in the model in order to have the same ratio between TBC and metal thickness. 
In order to consider the presence of TBC there are two approaches: to discretize the TBC including an extra mesh or to modify the thermal gradients at the interface between fluid and metal to take into account the presence of TBC. The presence of TBC has been taken into account by modifying the energy fluxes at the interface as shown in fig 13.

In Fig. 13 is shown the original formulation of the HybflowCHT code and how it has been modified in the present formulation. In order to model the thermal barrier coating (TBC) and the bond coat (BC) it is not necessary to mesh this region. An equivalent thermal resistance is introduced at the interface to model the presence of three layers with different materials.

Figure 14 shows the averaged distribution of non dimensional spanwise gradients over the cylinder with thermal barrier coating. In the top right corner there is a schematic of the cylinder with a red surface representing the coating.

Due to the presence of this layer of different conductivity two surfaces can be identified: the interface between metal and bond coat, the inner surface under the TBC, and the interface between thermal barrier coating and fluid, the outer surface over the TBC. The first surface shows the metal gradients that can generate structural cracks as highlight in [4]. The second surface is useful to identify problems such as excessive gradients in the ceramic surface that can induce spallation. The stainless steel solution is the continuous line the NiAl is the dashed line. For each material there are three lines. The lower one of each series represents the gradients on the inner

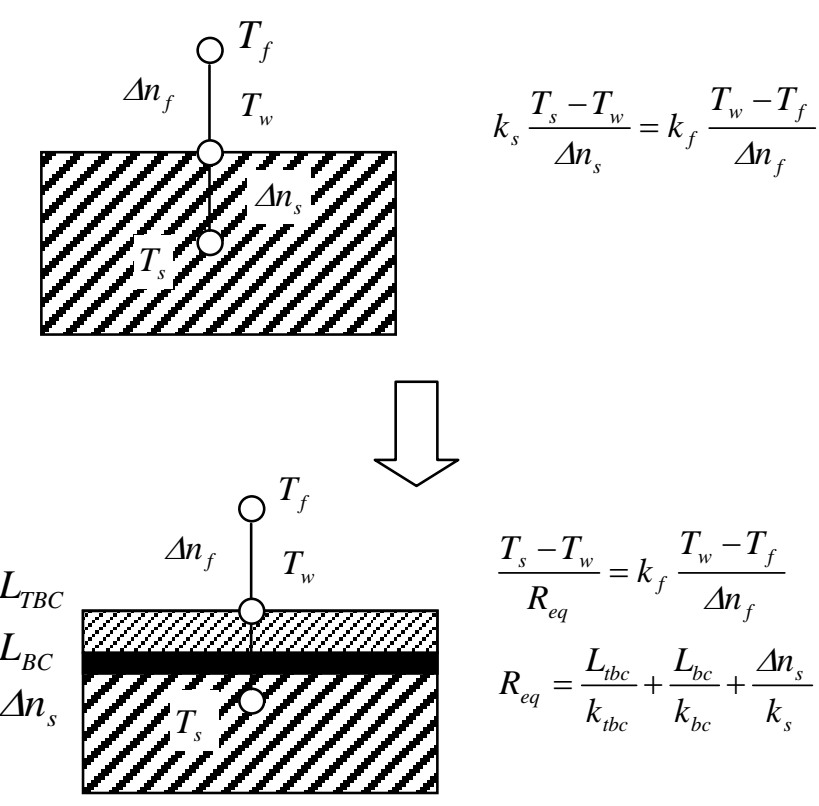

Fig. 13 energy flux formulation with thermal barrier coating 


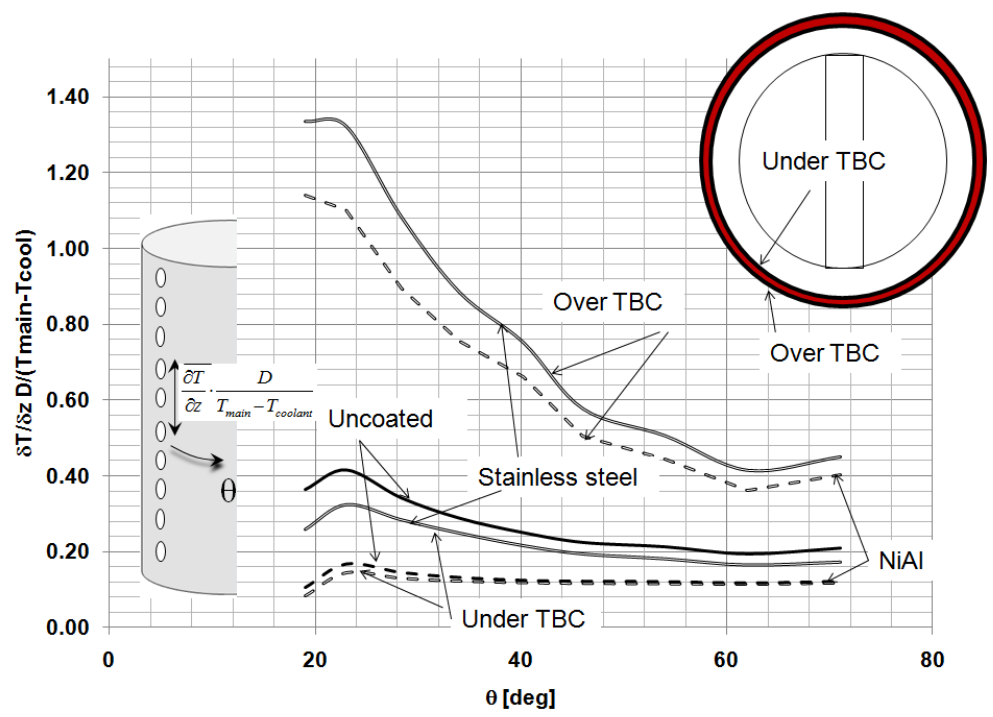

Fig. 14 mean spanwise NDG with TBC

surface. The upper line represents the gradients on the thermal barrier coatings. The middle line is the reference value without TBC. It is evident from the graph that by adding a coating the metal gradients are reduced. The positive impact of $\mathrm{NiAl}$ is still present with a reduction of metal gradients of about 3 times.

The beneficial effect of having a material with high thermal conductivity has an impact also on the outer surface. In particular the thermal stresses are reduced by about $20 \%$ with less risk of spallation near the coolant holes.

\section{CONCLUSIONS}

In this work the impact of conduction on thermal gradients in a cooled cylinder using a combination of impingement and film cooling is investigated. The experimental results comparing polycarbonate and stainless steel show that conduction has a strong impact on the overall level of effectiveness. The presence of impingement plate generates a region of high effectiveness located at $\theta=90$ degrees. In the case with stainless steel the level of effectiveness induced by the plate is very high.

Numerically the possibility of using even higher conductivity materials for the leading edge is studied. In particular a nickel alluminide alloy is modelled to evaluate the impact of a material with higher conductivity. A non-dimensional thermal gradient is defined to evaluate how the thermal gradients in the present test can impact a real gas turbine.

The results show that the thermal gradients near the coolant holes are from 3 to 4 times smaller if a nickel aluminide insert is used in the leading edge. By including the presence of thermal barrier coating in the simulation the interface between metal and bound coat shows a reduction of thermal gradients of about 3 times. Numerically it has been observed that even the gradient on the interface between TBC and fluid are reduced by about $20 \%$ reducing the risk of spallation. The use of high conductivity material seems a promising way to control and minimize thermal stresses near the coolant holes in gas turbine leading edges. 


\section{REFERENCES}

[1] Fadlun E. A., Michelizzi I. De laco M.: "Measurement error influence on gas turbine operability for condition-based maintenance and reliability/availability improvement", ASME Turbo Expo 2008, GT2008-50749

[2] Brandts D.E., Wesorick R.R.: "GE Gas turbine design philosophy, GE Industrial \& Power Systems", GER-3434D, 1994

[3] Tret'yachenko G. N., L. V. Kravchuk, V. A. Konev and R. I. Kuriat: "Resistance of marine gas turbine nozzle vanes to thermal cycling in a gas stream containing sea-water salts, Institute of Strength of Materials", Academy of Sciences of the Ukrainian SSR, Kiev., Problemy Prochnosti, No. 10, pp. 54-57, (translated in the Journal of Strength of Materials, Volume 6, Number 10, 1218-1220, 1974)

[4] Mazur Z., Hernandez-Rossette A., Garcia-Illescas R., Luna-Ramirez A., "Failure analysis of a gas turbine nozzle", Journal of Engineering Failure Analysis Vol. 15 (2008) 913-921

[5] Bunker R.S.: "Gas Turbine Heat Transfer: Ten Remaining Hot Gas Path Challenges", Journal of Turbomachinery, 2007, Vol. 129, pp 193-201

[6] Eifel M., Caspary V., Honen H., Jeschke P.: “Experimental and Numerical Analysis of Gas Turbine Blades With Different Internal Cooling Geometries", Journal of Turbomachinery, 2011, Vol. 133, pp 011018-1-9

[7] Verstraete T., Amaral S., Van de Braembussche, Arts T.: "Design and optimization of the Internal Cooling Channels of a High Pressure Turbine Blade - Part II: Optimization", Journal of Turbomachinery, 2010, Vol. 132, pp 021014-1-9

[8] Sellers, R. R., Soechting, F. O., Huber, F. W., and Auxier, T. A., 1998, "Cooled Blades for a Gas Turbine Engine," U.S. Patent No. 5,720,431.

[9] Dailey, G. M., McCall, R. A., and Evans, P. A., 2000, "Cooled Aerofoil for a Gas Turbine Engine," European Patent Application EP-1-022-432-A2. [8]

[10] Ekkad, S.V., Han, J.C., and Du, H., 1998, "Detailed Film Cooling Measurements on a Cylindrical Leading Edge Model: Effect of Free-Stream Turbulence and Coolant Density," Journal of Turbomachinery, Vol. 119, pp. 594-600.

[11] Gao Z., Wright L.M. , and Han J.C.: "Assessment of Steady State PSP and Transient IR Measurement Techniques for Leading Edge Film Cooling", Imeche conference 2005, IMECE200580146

[12] Coat A. and Lock G.D., "Flow visualisation experiments for turbine film cooling" , The Aeronautical Journal 2004, 403-409,

[13] Teng S., Sohn D.K., Han J-C: "Unsteady Wake Effect on Film Temperature and Effectiveness Distributions for a Gas Turbine Blade", Journal of Turbomachinery, 2000,Vol 122, pp 340-347 
[14] Ethridge M.I., Cutbirth M., Bogard D.G.: "Scaling of Performance for Varying Density Ratio Coolants on an Airfoil With Strong Curvature and Pressure Gradient Effects", Journal of Turbomachinery 2001, Vol 123, pp 231-237

[15] Adami, P., Martelli F. and Michelassi V., 2000, "Three-Dimensional Investigations for Axial Turbines by an Implicit Unstructured Multi-block Flow Solver", ASME, IGTI TurboExpo 2000.

[16] Montomoli F., Adami P., Martelli F. "A finite volume method for the conjugate heat transfer in film cooling devices" Journal of Power and Energy, Proceedings of the Institution of Mechanical Engineers, Part A 2009, 223(A2), 191-200

[17] Barth T.J. "Numerical Aspects of Computing Viscous High-Reynolds Number Flows on Unstructured Meshes", AIAA Paper 91-0721, Jan. 1991.

[18] Wilcox D.C. "Turbulence modeling for CFD” DWC Industries, Inc., La Canada, 1993

[19] Harrison K. and Bogard D.: "Comparison of RANS Turbulence Models for Prediction of Film Cooling Performance", ASME Turbo Expo 2008, GT2008-51423

[20] Montomoli F., Massini M., Adami P., Martelli F.: "Effect of Incidence Angle with Wake Passing on a Film Cooled Leading Edge: a Numerical Study", International Journal for Numerical Methods in Fluids, Wiley, 2009, Vol. 63, Issue 12, 1359-1374

[21] Montomoli F., Massini M, Salvadori S., Martelli F: "Geometrical Uncertainty and Film Cooling: Fillet Radii", Journal of Turbomachinery, 2011, Vol. 134

[22] Verstraete T., "Multidisciplinary Turbomachinery Component Optimization Considering Performance, Stress and Internal Heat Transfer", PhD thesis, Von Karman Institute for Fluid Dynamics-Universiteit Gent, 2008

[23] Gao Z., Yang H., Wright L., Han J-C, "Conjugate Prediction of Leading Edge Film Cooling and Heat Transfer", 2006, ISROMAC11-2006-45

[24] Chernobrovkin A.A. and Lakshminarayana A., "Numerical Simulation of Complex Turbomachinery Flows", 1999, NASA /CR-1999-209303

[25] Kline, S. J., and F. A. McClintock: "Describing Uncertainties in Single-Sample Experiments", Mech. Eng., p. 3, January 1953

[26] Luikov, A. V. Conjugate convective heat transfer problems. International J. HeatMass Transf., $1974,17,1207-1214$.

[27] Martiny, M., Schiele, R., Gritsch, M., Schulz, A., and Kim, S. High efficient cooling concepts for low emission combustors. In High intensity combustors-steady isobaric combustion (Eds S. Wittig, $\mathrm{O}$. Vöhringer, and S. Kim), 1998, pp. 261-281 (Wiley-VCHVerlagGmbH\&Co.KGaA).

[28] Maikell J., Bogard D., Piggush J, Kohli A.: "Experimental Simulation of a Film Cooled Turbine Blade Leading Edge Including Thermal Barrier Coating Effects", Journal of Turbomachinery, Vol. 133 / 011014-1-7 
[29] Bogaard R. H., in Thermal Conductivity 18, Proc. 18th Int. Conf. on Thermal Conductivity, T. Ashworth and D. R. Smith, eds. (Plenum, New York, 1985), pp. 175-185.

[30] Chu T. K. and Ho C. Y., in Thermal Conductivity 15, Proc. 15th Int. Conf. on Thermal Conductivity, V. Mirkovich, ed. (Plenum, New York, 1977), pp. 79-104.

[31] P. A. Durbin. "On the k- $\varepsilon$ stagnation point anomaly", International Journal of Heat and Fluid Flow, 17(1): 89-90, 1996

[33] Kline, S. J., and F. A. McClintock: "Describing Uncertainties in Single-Sample Experiments", Mech. Eng., p. 3, January 1953 